\title{
Experimental reconsideration of the utility of serum starvation as a method for synchronizing mammalian cells
}

\author{
Stephen Cooper*, Mariam Gonzalez-Hernandez ${ }^{1}$ \\ Department of Microbiology and Immunology, University of Michigan Medical School, Ann Arbor, MI 48109-0620, USA
}

Received 2 September 2008; accepted 22 September 2008

\begin{abstract}
Accurate cell-size determinations support the prediction that serum starvation and related whole-culture methods cannot synchronize cells. Theoretical considerations predict that whole-culture methods of synchronization cannot synchronize cells. Upon serum starvation, the fraction of cells with a G1-phase amount of DNA increased, but the cell-size distribution is not narrowed. In true synchronization, the cell-size distribution should be narrower than the cell-size distribution of the original culture. In contrast, cells produced by a selective (i.e. non-wholeculture) method have a specific DNA content, a narrow size distribution, and divide synchronously. The general theory leading to the conclusion that whole-culture methods for synchronization do not work implies that one can generalize these serum-starvation results to other cell lines and other whole-culture methods, to conclude that these methods do not synchronize cells.
\end{abstract}

(C) 2008 International Federation for Cell Biology. Published by Elsevier Ltd. All rights reserved.

Keywords: Serum starvation; Synchronization; Cell cycle; G1 phase; Membrane elution

\section{Introduction}

To understand the passage of a cell through the cell cycle, it is important to know what events take place at different times during the cell cycle. Because the measurement of chemical events is difficult in single cells, much effort has been expended on methods to synchronize cells (Davis et al., 2001). A well-synchronized culture is one in which the cells move as a uniform cohort through the cell cycle; truly synchronized cells should accurately reflect the events occurring in unperturbed cells during normal passage through the cell cycle. The sine qua non of synchronization is that the cells move uniformly through the cell cycle and divide synchronously over a relatively narrow span of time (Cooper, 2004a; Cooper and Shedden, 2003).

Synchronization methods can be separated into two classes, whole-culture methods and selective methods (Cooper, 1991).

\footnotetext{
* Corresponding author. Tel.: +1 734764 4215; fax: +1 7347643562.

E-mail address: cooper@umich.edu (S. Cooper).

${ }^{1}$ Present address: Pontifical Catholic University of Puerto Rico, Ponce, PR 00717.
}

Whole-culture methods are those in which treatment of all cells in a culture is proposed to lead to a group of cells that are arrested at a specific point in the cell cycle, and release of the arrested cells is presumed to produce a synchronized culture. Selective methods, in contrast, are methods in which a subset of the original population is selected to produce a synchronized culture and the remaining cells are discarded.

It is important to distinguish between cells that merely have one property in common (e.g. all cells have a G1-phase amount of DNA), and cells that are truly synchronized, where the cells have all cellular properties in common and are reflective of a particular cell-cycle age. Cells that are not synchronized but have one property in common should be referred to as 'aligned' for a particular property, and not as 'synchronized'. Gong et al. (1995) found that 'chemically' treated cells exhibit growth imbalance and higher heterogeneity with respect to all parameters measured, including the expression of cyclins and the degree of pRB phosphorylation.

What is not taken into account by whole-culture synchronization methods is the generality of the evidence that wholeculture methods cannot synchronize cells (Cooper, 2003b). This does not imply that whole-culture methods synchronize 
cells poorly, or weakly, or only occasionally. Rather, it proposes that such methods do not synchronize cells at all (Cooper, 2003b), but merely align cells for some particular property (e.g. DNA content).

The importance of the proposal that whole-culture synchronization methods do not work, when clearly stated (i.e. serum starvation does not synchronize cells) is that widely used methods of studying the cell cycle fail to yield information regarding the normal cell cycle. Observations of cyclical patterns and other changes following whole-culture synchronization are artifacts of the synchronizing treatment (Shedden and Cooper, 2002a,b).

Recent experiments support the theoretical critique of whole-culture methods. A study of lovastatin, a compound that was originally proposed to be a general whole-culture synchronizing agent (Keyomarsi et al., 1991), demonstrated that lovastatin does not synchronize cells (Cooper, 2002). Time-lapse analysis of cell division following lovastatin arrest and release has shown that the cells do not divide synchronously (Cooper, 2002). Further, reconsideration of the published data showing synchronized S phases and initial arrest with a G1-phase amount of DNA demonstrated that the S phases are not synchronized, and that the cells are not even arrested with a G1-phase amount of DNA (Cooper, 2002).

Another experimental example of problems with wholeculture synchronization is the use of nocodazole. Nocodazole is widely used as a synchronizing agent (see, for example, Harper, 2005; Jansen-Durr et al., 1993; Laoukili et al., 2008; Ludlow et al., 1993; Ouyang et al., 1998). Cells treated with nocodazole are arrested prior to mitosis and are aligned with a G2 amount of DNA. Support for this proposal is the widely reported observation that nocodazole treatment produces cells with a G2-phase amount of DNA (Cooper et al., 2006; Kung et al., 1990). Experiments have demonstrated that even when a culture inhibited with nocodazole has essentially all cells with a G2-phase amount of DNA, these cells are not synchronized (Cooper et al., 2006). The two criteria upon which this conclusion was based are the observations that there was no narrowing of the cell-size distribution after inhibition with nocodazole, and that there is no synchronized cell division following release from nocodazole inhibition (Cooper et al., 2006).

Thymidine block, either as a single or a double block, has also been used to synchronize cells (Bar-Joseph et al., 2008). A reevaluation of the data from a microarray study of gene expression during the cell cycle of human cells (Cho et al., 2001) has led to the conclusion that the double-thymidine block used in this study did not synchronize cells (Shedden and Cooper, 2002a). A more direct test of the use of thymidine block to synchronize cells has shown that this whole-culture method does not synchronize cells (Cooper et al., 2008).

We now turn to examine one of the most widely used methods to 'synchronize' cells, serum starvation. This method has been used in hundreds or even thousands of papers to study the events during the cell cycle (for recent examples, see Bar-Joseph et al., 2008; Le Francois et al., 2007). When cells are placed in low serum concentrations, it is generally observed that the fraction of cells with a G1-phase amount of DNA increases. It was concluded that these cells are 'arrested at a point in the G1 phase'. When normal serum concentrations are restored, it is proposed that the cells move synchronously through the cell cycle. Serum starvation has been analyzed as a synchronizing method and it was shown that serum starvation does not synchronize cells (Liliensiek et al., 2006). In these experiments, when cells were serumstarved between 24 and $96 \mathrm{~h}$ the increase in the proportion of cells with a G1-phase amount of DNA was negligible. Of course this result can be criticized as the cells used did not achieve a G1-phase amount of DNA.

The question arises as to how many experiments, or results, or critical tests, must be presented to show that a method used by an enormous number of laboratories does not work. Does one have to test every cell line that was used in a serumstarvation experiment, and test every protocol used to synchronize cells by using low serum concentrations? If a single experiment were presented that showed the method did not work, such an experiment could be criticized as 'anecdotal', and not a proof that the method does not work. In more general terms, it may impossible to present an experimental proof that some method or protocol does not work. More generally, it is proposed that one cannot have an experimental proof of a universal negative.

So how does one show that the widely used serum starvation method does not work? What one must do is combine a general theoretical proposal with an experiment that supports that theoretical proposal. Here we show that the predictions of a theoretical analysis of serum starvation are fulfilled when the cell-size distribution of serum-starved cells is compared to cells growing in normal concentrations of serum. The introduction of a sensitive cell-size measuring method, when applied to the problem of whole-culture synchronization, clearly show that one of the key criteria of a synchronized culture, that the cell-size distribution of the synchronized cells must be narrower than the original cells, is not fulfilled when cells are grown in low concentrations in serum.

We present a simple set of experiments using improved cell-size determinations to analyze the use of serum starvation or serum deprivation to produce 'synchronized' cells. We have studied the effect of incubation in low serum concentrations on L1210 mouse cells. We have measured the size distribution of these cells, and observe that there is no narrowing of the cellsize distribution. This result is strengthened when the serum starvation result is compared to cells produced by a selective method of synchronization. Comparison of DNA and size measurements of serum-starved cells with cells produced by a selective method (membrane elution) support the proposal that serum-starved cells do not have a narrowed size distribution and therefore are not synchronized.

Possibly more important than this particular result, we propose that the results presented here are not restricted to the cells and conditions used in this study. Rather, these results apply broadly and generally to mammalian cells and other growth systems and, of course, other whole-culture methods of synchronization. It might be suggested that the results 
presented here are applicable only to the particular cell line studied, and cannot be extended to other cell lines. This viewpoint suggests that in order to prove that serum starvation cannot synchronize cells one must look at essentially every (or perhaps the majority) of cell lines and show that this treatment does not synchronize any of these cells. However, the experiments presented here are strongly buttressed by theoretical considerations rigorously supporting the proposal that wholeculture methods cannot synchronize cells (Cooper, 1998b; Cooper, 2003b,c, 2004a,c, 2006; Cooper and Shedden, 2003). The prohibition against the experimental demonstration of a universal negative, viz., that whole-culture methods cannot synchronize cells, therefore does not apply in this case.

We propose that the fit of theory and experiment allows the extension of these results to other cell lines. To the theoretical concept that these whole-culture methods 'cannot synchronize cells', we present a simple experimental result supporting the proposal that whole-culture methods 'do not synchronize cells'.

\section{Materials and methods}

\subsection{Cells}

L1210 cells, a mouse leukemic line (ATCC designation CCL219) are used for all experiments. These cells are nonadherent and grow with a doubling time of 9-10 h.

\subsection{Growth media}

Liebovitz's L-15 medium (cellgro by Mediatech, Herndon, VA 20171) was supplemented with $2 \mathrm{mg} / \mathrm{ml}$ glucose, $100 \mathrm{U} / \mathrm{ml}$ penicillin, $100 \mu \mathrm{g} / \mathrm{ml}$ streptomycin, and 10\% Cosmic Calf Serum. Cosmic Calf Serum (CCS) prepared by Hyclone, Inc., is a modified calf serum that substitutes for fetal bovine serum (FBS). The cost of CCS is between one-fifth and one-tenth that of FBS. The buffering in $\mathrm{L}-15$ medium allows cell growth and $\mathrm{pH}$ maintenance without a $\mathrm{CO}_{2}$ atmosphere. Cells were grown at $37^{\circ} \mathrm{C}$ in sealed flasks. Cells were kept below 200,000 cells/ml during exponential growth. Under these growth conditions, the cells were in steadystate growth and did not approach overgrowth conditions.

\subsection{DNA analysis}

Cells were collected by centrifugation, washed once in phosphate-buffered saline (PBS, pH 7.4), and suspended in 70\% ethanol. After ethanol treatment in the cold for at least $20 \mathrm{~min}$, the cells were collected by centrifugation, the ethanol was aspirated off, and the cells were suspended in PBS containing propidium iodide $(50 \mu \mathrm{g} / \mathrm{ml})$ and RNase A $(100 \mu \mathrm{g} / \mathrm{ml})$. After at least $20 \mathrm{~min}$ incubation on ice, the cells were analyzed in a Becton-Dickinson FACScan analyzer by using Cellquest software. Further analysis of the data was carried out with WINMdi software.

\subsection{Cell counting and cell sizing}

Cells were counted and sized by using a Coulter Z2 electronic cell counter with a $70-\mu \mathrm{m}$ orifice. The data were collected and analyzed with the Z2 AccuComp program from Beckman Coulter (version 3.01). Cells were counted directly in L-15 medium. Analyses of the size distributions were performed by using an Excel spreadsheet program.

\subsection{Membrane elution}

The membrane-elution apparatus and method has been colloquially referred to as the 'baby machine', as it continuously produces newborn cells. This apparatus has been described in detail before (Eward et al., 2004; Helmstetter et al., 2003; Thornton et al., 2002), but some of the details will be presented here. A support screen (Millipore, catalogue number YY3014234) was secured in a holder with rubber gaskets so that a membrane (Millipore catalogue number GSWP14250; $142-\mathrm{mm}$ nitrocellulose membrane, $0.22-\mu \mathrm{m}$ pores) lay directly on the support screen. A Lucite ring confined the liquid to the top of the membrane. Rubber gaskets between the membrane and Lucite ring prevented leakage. The support screen was atop a funnel that could be inserted into a side-arm flask to allow suction to pull the medium through the membrane.

Cells were grown to a concentration of $<200,000$ cells $/ \mathrm{ml}$ to obtain $\sim 60-70$ million cells. All experiments were carried out in a warm room. The membrane holder was also in a fullview incubator within the warm room to ensure constant temperature. To start the production of newborn cells, $50 \mathrm{ml}$ PBS with $10 \mu \mathrm{g} / \mathrm{ml}$ concanavalin A at $37^{\circ} \mathrm{C}$ was filtered through the membrane until no residual liquid remained. Warm PBS $(100 \mathrm{ml})$ was filtered through the membrane to remove unbound concanavalin $\mathrm{A}$; again, no residual liquid remained. Cells in 300-600 $\mathrm{ml}$ of medium were slowly poured on to the membrane with gentle suction over 3-5 min. When $20-30 \mathrm{ml}$ of liquid remained above the membrane, the liquid was poured off so that the cells were never dried and exposed to air. The membrane apparatus was inverted and filled with fresh medium. Medium from a reservoir was pumped through the membrane. After 5-10 min the unbound and weakly bound cells had been removed. The cells obtained from this initial flow of medium through the membrane (along with the cells in the residual medium) were collectively referred to as the 'wash-off'. The wash-off was on the order of $10-20 \%$ of the input cells. This means that over $80 \%$ of the initial cells remained bound to the membrane. Fresh medium was pumped into the membrane holder at a rate of $2.0-3.0 \mathrm{ml} /$ min. The cells obtained by elution from the membrane are termed newborn or 'baby' cells.

\section{Results}

\subsection{DNA distribution following incubation in low concentrations of serum}

Exponentially growing L1210 cells were collected by centrifugation and suspended in different but low concentrations of serum. We explored the result of incubating cells from 20 to $96 \mathrm{~h}$ with serum concentrations between 0 and $3.0 \%$. 
This is equivalent to determining the best conditions to 'synchronize' cells. This exploration led to the identification of conditions that produce cells with an elevated fraction of cells with a G1-phase amount of DNA. An example of the increase in cells with a G1-phase amount of DNA is shown in Fig. 1. The growth-arrested population did not have all cells with a G1-phase amount of DNA. Rather, there was an increase in the fraction of cells with a G1-phase amount of DNA. This result is commonly observed with serum starvation.

\subsection{Size distribution of serum starved cells}

The size distribution of serum-starved cells was smaller than for the exponential cells, and the cells had a broad cellsize distribution (Fig. 2). The cell-size distribution is inconsistent with the sizes of cells that are truly synchronized. For comparison, cells produced by membrane elution without any inhibition of growth show that the size distribution of newborn cells is consistent with the distribution expected from a truly synchronized culture (Fig. 3). As discussed in detail below, the criterion of requiring a narrow size distribution for true synchronization indicates that serum- starved cells were not perforce synchronized and do not represent cells of a particular age during the normal cell cycle.

\section{Discussion}

\subsection{The purpose of synchronization}

The purpose of synchronizing cells is to produce cells that can be used to study and understand events occurring during the normal, unperturbed cell cycle. A truly synchronized culture is one that mimics or reproduces the passage of cells through the normal cell cycle. A newborn cell has a number of different properties (e.g. DNA content, cell size, protein

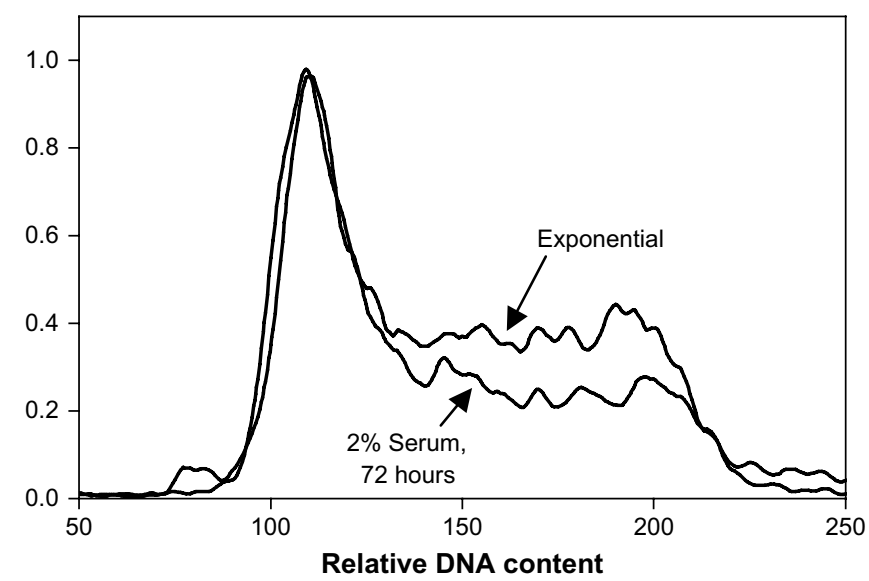

Fig. 1. DNA content of serum- deprived cells compared with exponential cells. The DNA distributions were determined as described in the methods and the patterns are plotted using an excel spreadsheet. The thick line is the DNA distribution of cells grown for $72 \mathrm{~h}$ at a concentration of $2.0 \%$ CCS. There is an increase in the fraction of cells with a G1-phase amount of DNA in the serum-deprived cells.

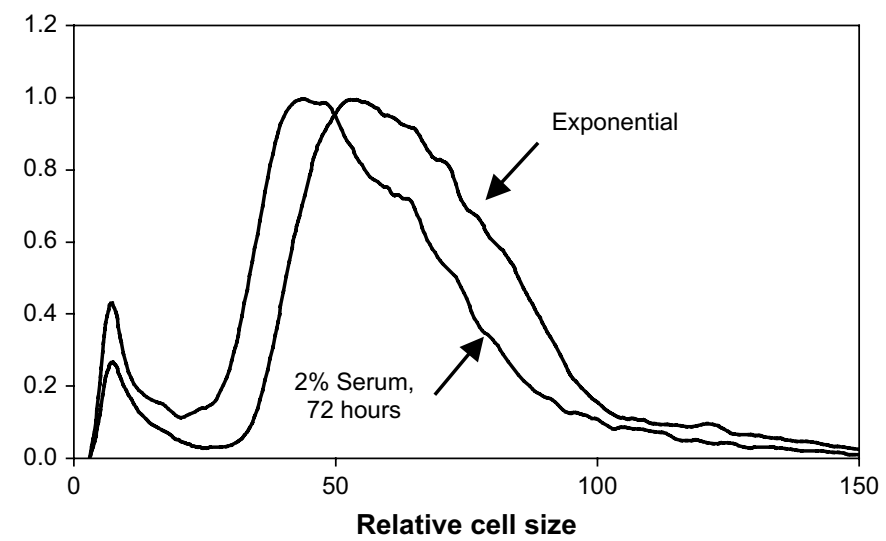

Fig. 2. Size distribution of serum- deprived cells compared with exponential cells. The size distribution of L1210 cells incubated for $72 \mathrm{~h}$ in $2.0 \% \mathrm{CCS}$ are compared with cells grown in 10\% CCS. These are the same cells as in Fig. 1. The cell-size distribution of the starved cells is as broad as that for newborn cells. Furthermore, the serum- deprived cells are smaller than the exponentially growing cells. There is no narrowing of the size distribution as would be expected for truly synchronized cells.

composition, internal cellular arrangement). As a cell passes through the cell cycle, each of these must necessarily change in order to produce a dividing cell at the end of the cell cycle. At a minimum, each component of the cell must double so that the cell about to divide has twice as much of everything in the newborn cell. At each time point during the cell cycle, a cell has a particular constellation of properties that are on the trajectory from newborn cell to dividing cell. A synchronized

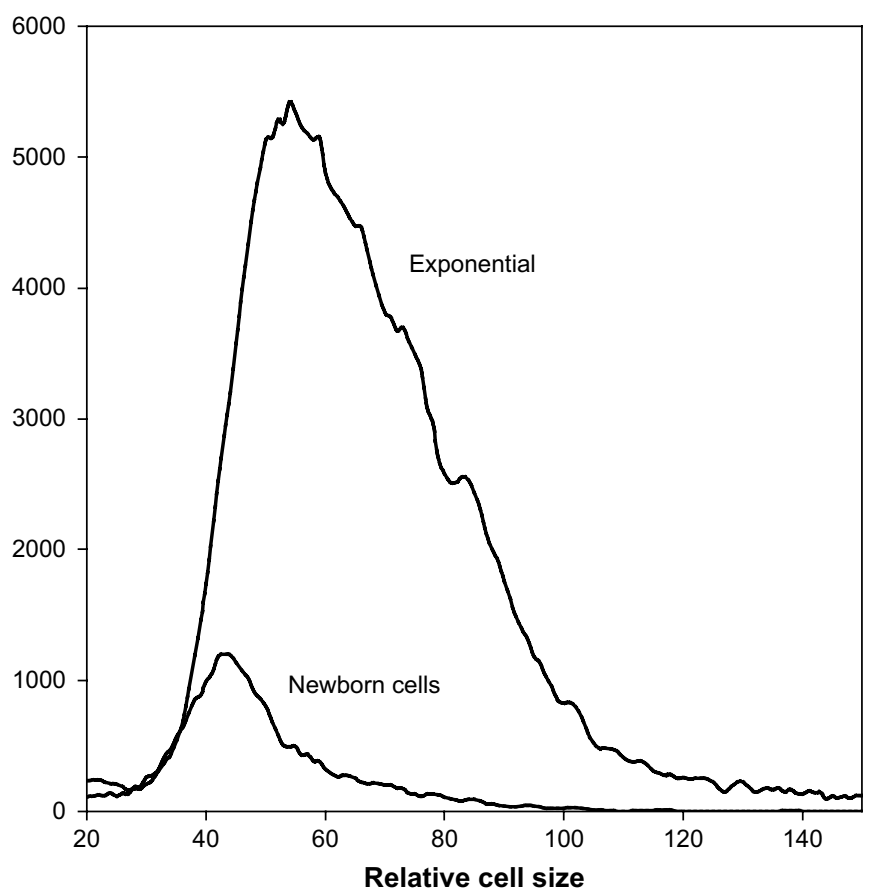

Fig. 3. Size distribution of newborn cells produced by membrane elution. Newborn cells are compared with exponentially grown cells. The newborn cell-size distribution fits into the lower size distribution of the exponential culture, as would be expected of newborn cells. Further, the size distribution of newborn cells is narrower than exponentially grown cells. 
culture should, at each point during passage through the cell cycle, have cells with the specific properties associated with each cell-cycle age during the growth of an unperturbed cell. Thus, if we consider two properties of a growing cell, e.g. cell size and DNA content, a newborn cell of age 0 has a size of 1.0 and a DNA content of the G1 phase. Size usually increases continuously during the cell cycle (Cooper, 1998a) so that at division (age 1), the cell-size is 2. DNA also increases during the cell cycle so that dividing cells have a DNA content that reflects the G2-phase amount of DNA. Just prior to division, e.g. at age 0.8 , a cell may have a G2-phase amount of DNA, and the cell size could be approximately size 1.8. If a synchronized culture truly reflected the normal cell cycle, one would expect to find that cells arrested with a G1-phase amount of DNA would have a relatively narrow cell-size distribution of about size 1.1. With biological and statistical variation considered, the size distribution could vary from 0.9 to 1.2 , or even be as wide as 0.8 to 1.4 . But in any case, the size distribution should not be as wide as the exponential culture.

To summarize, the size distribution of cells at any time in a truly synchronized culture has to be narrower than the size distribution of the original culture. If the cell-size distribution in the treated cells is as wide or wider than the exponential cell-size distribution, this would indicate that the cells were not synchronized and did not reflect the normal cell size at any point during the division cycle. Such unsynchronized cells are presumably arrested with only one particular property common to all cells. Such cells would be 'aligned' for that particular property. In the case of serum starvation, this property is an increase in cells with a G1-phase amount of DNA. Other properties would be reflective of, or associated with, cells of all ages, as the size distribution in this case would include cells of all different cell-cycle sizes.

\section{2. 'Synchronization' with serum starvation or serum deprivation}

The experimental results indicate that while incubation of cells in low concentrations of serum produces an L1210 cell population with increased proportion of cells with a G1-phase amount of DNA, these cells are not synchronized because the cell-size distribution of inhibited cells had not narrowed. Most importantly, these serum deprivation experiments support the theoretical predictions that whole-culture methods cannot synchronize cells (Cooper, 1998b, 2003b, 2004a,b; Cooper and Shedden, 2003). Thus, while the cell-size distribution is not narrowed, the entire size distribution shifts to smaller cell sizes, which also supports the theoretical considerations of serum starvation (Cooper, 2003b).

\subsection{An explanation for the increase in cells with a G1- phase amount of DNA during serum deprivation}

One of the major generalizations of studies of the effect of change in interdivision time on the length of the G1, S, and G2 phases is that the $\mathrm{S}$ and $\mathrm{G} 2$ phases are relatively invariant and that the most variable phase is the G1 phase of the cell cycle (Helmstetter et al., 1968; Cooper, 1979). To give a numerical example, if a cell with a 20 -h interdivision time had cells with a 10 -h G1 phase, a 5 -h $\mathrm{S}$ phase and a 5 -h G2 phase, there would be $\sim 50 \%$ of the cells with a G1-phase amount of DNA. If these same cells were now placed in conditions that produced a 100- $\mathrm{h}$ interdivision time, the G1 phase would occupy $90 \mathrm{~h}$ if $\mathrm{S}$ and $\mathrm{G} 2$ phases were truly invariant and $\sim 90 \%$ of the cells would have a G1-phase amount of DNA. Yet the crux here is that these slower growing cells would still be growing, and not arrested with a G1-phase amount of DNA. Rather, there would be a simple increase in the fraction of cells with a G1-phase amount of DNA without cells being arrested at any point in the G1 phase, as has become generally assumed.

The change in cell-cycle analysis from methods that give absolute times of the phases (such as with frequency of labeled mitoses (FLM)) to methods that merely determine fractions of cells with different DNA contents (FACS) has led to the loss measurements of the time that cells are in different phases (Cooper, 2003a). We postulate that when cells are placed in low concentrations of serum the cells are not totally arrested in growth but grow slowly with a much longer interdivision time. (In many 'serum-starvation' experiments, the cells are not really starved, but merely placed in a low concentration of serum $(\sim 0.1-2 \%)$, and this could lead to growth at a very slow rate.) This leads to a larger fraction of cells with a G1-phase amount of DNA. There is no need to postulate an arrest state such as proposed for a restriction point (Pardee, 1974), or an out of phase cell position as postulated for G0, or any conclusion that cells exit the cell cycle (Cooper, 2003c).

\subsection{Explanation of decrease in cell size during serum deprivation}

When cells are placed in growth deficient media, the decrease in the rate of mass increase leads to a decrease in the rate of initiations of $\mathrm{S}$ phase. However, cells that were in $\mathrm{S}$ and $\mathrm{G} 2$ at the time of medium change continue through these phases and divide in a relatively normal pattern. This means that for a period of time cell number increases faster than mass increase. The average cell mass is the ratio of the total cell mass divided by the number of cells. As cell numbers increase faster than cell mass for a period of time during the transition to slower growth medium (low serum), the cell size would be expected to decrease (Cooper, 2003b). Thus, the finding that cell size is smaller during growth in low serum is consistent with the theoretical predictions.

\subsection{Published work on synchronization by serum starvation}

The literature on the use of low concentrations of serum to induce synchronization is now enormous, making it impossible to review them comprehensively, but a few examples can be noted here. Liliensiek et al. (2006) clearly conclude that 
serum starvation does not synchronize cells. However, Di Matteo et al. (1995) claim that serum starvation $(36 \mathrm{~h})$ did synchronize cells, but an examination of their as published results of DNA distributions during growth after re-feeding serum indicated that the cells were clearly not synchronized (Cooper, 1998b). A recent example of serum starvation used to produce a synchronized culture also shows that the cells are not synchronized (Bar-Joseph et al., 2008). While cells starved of serum may have more cells with a G1-phase amount of DNA, when the cells are released from starvation there is no indication that the cells move progressively through the cell cycle. There is never a peak in cells with an S-phase amount of DNA followed by a peak of cells with a G2-phase amount of DNA. Further, cells with a G1-phase amount of DNA are always present at elevated amounts.

Perhaps the most important example of serum starvation is related to the proposal that cells are arrested at a particular point in the cell cycle described as the 'restriction point' (Pardee, 1974). Although it is widely believed that such cells are arrested at some specific point in the G1-phase of the cell cycle, it was noted by Pardee that 'different cells begin thymidine incorporation at different times. Thus, measurement of the time of initiation of DNA synthesis by a cell population depends upon the behavior of an early initiating subclass of the population'. This result means that upon release from serum-deprivation arrest, the cells are not synchronized and do not start DNA synthesis in a synchronous manner. A detailed critique of the restriction point proposal has been published (Cooper, 2003c).

The conclusion of this brief summary of work with serum deprivation as a synchronizing agent indicates that serum starvation does not synchronize cells since, upon replenishment of serum, these starved cells do not move as a synchronized cohort through the cell cycle.

\subsection{Generalization of results to other cell lines and growth conditions}

One critique of the extension of the results presented here to other cells and growth conditions might be that our results are merely related to the particular cells and growth conditions that we have used, viz. L1210 cells grown in L-15 medium with glucose (the cells and conditions used here). However, this proposal ignores the theoretical generalization that led to the experiments presented here. Theory predicts the results obtained here - that serum starvation does not synchronize cells - and the theory is independent of cell type or cell line (Cooper, 1998b, 2003b, 2004a,b; Cooper and Shedden, 2003).

Rather than placing the burden of proof on the proposal that all cell lines cannot be synchronized by whole-culture methods, we now need to shift the burden of proof to those who propose using such whole-culture procedures - including serum starvation - as 'synchronization' methods. We do not accept the proposal that perhaps 'just around the corner' there exists a cell line or a culture condition situation that permits synchrony to arise by these whole-culture methods.

\subsection{The simple message regarding serum starvation to synchronize cells}

The stark message is that serum deprivation does not synchronize cells. This result accords with theoretical considerations. It is important not to confuse this result with discussions about the way the cell passes through the cell cycle. The single question answered here is whether incubation in low serum can synchronize cells, to which the answer is a negative.

\section{Acknowledgements}

This work was supported by the National Science Foundation (grant MCB-0323346) and (in part) by the National Institutes of Health (University of Michigan's Cancer Center, support grant 5 P30 CA46592). M. G-H is associated with and supported by the Summer Research Opportunity Program (SROP) of the University of Michigan, which also added support to this research. Alexandra Cooper was invaluable as an editor of this paper.

\section{References}

Bar-Joseph Z, Siegfried Z, Brandeis M, Brors B, Lu Y, Eils R, Dynlacht BD, Simon I. Genome-wide transcriptional analysis of the human cell cycle identifies genes differentially regulated in normal and cancer cells. Proc Natl Acad Sci U S A 2008;105:955-60.

Cho RJ, Huang M, Campbell MJ, Dong H, Steinmetz L, Sapinoso L, Hampton G, Elledge SJ, Davis RW, Lockhart DJ. Transcriptional regulation and function during the human cell cycle. Nat Genet 2001;27:48-54.

Cooper S. A unifying model for the $\mathrm{G} 1$ period in prokaryotes and eukaryotes. Nature 1979;280:17-9.

Cooper S. Bacterial growth and division. San Diego, CA: Academic Press; 1991.

Cooper S. Length extension in growing yeast: is growth exponential? - yes. Microbiology 1998a;144:263-5.

Cooper S. Mammalian cells are not synchronized in G1-phase by starvation or inhibition: considerations of the fundamental concept of G1-phase synchronization. Cell Prolif 1998b;31:9-16.

Cooper S. Reappraisal of G1-phase arrest and synchronization by lovastatin. Cell Biol Int 2002;26:715-27.

Cooper S. How the change from FLM to FACS affected our understanding of the G1 phase of the cell cycle. Cell Cycle 2003a;2:157-9.

Cooper S. Rethinking synchronization of mammalian cells for cell-cycle analysis. Cell Mol Life Sci 2003b;6:1099-106.

Cooper S. Reappraisal of serum starvation, the restriction point, G0, and G1phase arrest points. FASEB J 2003c;17:333-40.

Cooper S. Is whole-culture synchronization biology's 'Perpetual Motion Machine'? Trends Biotechnol 2004a;26:266-9.

Cooper S. Rejoinder: whole-culture synchronization cannot, and does not, synchronize cells. Trends Biotechnol 2004b;22:274-6.

Cooper S. Whole-culture synchronization cannot, and does not, synchronize cells. Trends Biotechnol 2004c;22:274-6.

Cooper S. Bacterial and eukaryotic checkpoints and restriction points. Bioessays 2006;28:1035-9.

Cooper S, Shedden K. Microarray analysis of gene expression during the cell cycle. Cell Chromosome 2003;2:1-12.

Cooper S, Iyer G, Tarquini M, Bissett P. Nocodazole does not synchronize cells: implications for cell-cycle control and whole-culture synchronization. Cell Tissue Res 2006;324:237-42.

Cooper S, Chen KZ, Ravi S. Thymidine block does not synchronize L1210 mouse leukaemic cells: implications for cell cycle control, cell cycle 
analysis and whole-culture synchronization. Cell Prolif 2008;41:15667.

Davis PK, Ho A, Dowdy SF. Biological methods for cell-cycle synchronization of mammalian cells. Biotechniques 2001;30:1322-6. 1328, 1330-31.

Di Matteo G, Fuschi P, Zerfass K, Moretti S, Ricordy R, Cenciarelli C, Tripodi M, Jansen-Durr P, Lavia P. Transcriptional control of the Htf9A/RanBP-1 gene during the cell cycle. Cell Growth Differ 1995;6:1213-24.

Eward KL, Van Ert MN, Thornton M, Helmstetter CE. Cyclin mRNA stability does not vary during the cell cycle. Cell Cycle 2004;3:1057-61.

Gong J, Traganos F, Darzynkiewicz Z. Growth imbalance and altered expression of cyclins B1, A, E, and D3 in MOLT-4 cells synchronized in the cell cycle by inhibitors of DNA replication. Cell Growth Differ 1995;6:1485-93.

Harper JV. Synchronization of cell populations in G1/S and G2/M phases of the cell cycle. Methods Mol Biol 2005;296:157-66.

Helmstetter C, Cooper S, Pierucci O, Revelas E. On the bacterial life sequence. Cold Spring Harb Symp Quant Biol 1968;33:809-22.

Helmstetter CE, Thornton M, Romero A, Eward KL. Synchrony in human, mouse and bacterial cell cultures - a comparison. Cell Cycle 2003;2:42-5.

Jansen-Durr P, Meichle A, Steiner P, Pagano M, Finke K, Botz J, Wessbecher J, Draetta G, Eilers M. Differential modulation of cyclin gene expression by MYC. Proc Natl Acad Sci U S A 1993;90:3685-9.

Keyomarsi K, Sandoval L, Band V, Pardee AB. Synchronization of tumor and normal cells from G1 to multiple cell cycles by lovastatin. Cancer Res 1991;51:3602-9.

Kung AL, Sherwood SW, Schimke RT. Cell line-specific differences in the control of cell cycle progression in the absence of mitosis. Proc Natl Acad Sci U S A 1990;87:9553-7.
Laoukili J, Alvarez M, Meijer LA, Stahl M, Mohammed S, Kleij L, Heck AJ, Medema RH. Activation of FoxM1 during G2 requires cyclin A/Cdkdependent relief of autorepression by the FoxM1 N-terminal domain. Mol Cell Biol 2008;28:3076-87.

Le Francois BG, Maroun JA, Birnboim HC. Expression of thymidylate synthase in human cells is an early $\mathrm{G}(1)$ event regulated by CDK4 and p16INK4A but not E2F. Br J Cancer 2007;97:1242-50.

Liliensiek SJ, Schell K, Howard E, Nealey P, Murphy CJ. Cell sorting but not serum starvation is effective for SV40 human corneal epithelial cell cycle synchronization. Exp Eye Res 2006;83:61-8.

Ludlow JW, Glendening CL, Livingston DM, DeCaprio JA. Specific enzymatic dephosphorylation of the retinoblastoma protein. Mol Cell Biol 1993;13:367-72.

Ouyang B, Lan Z, Meadows J, Pan H, Fukasawa K, Li W, Dai W. Human Bub1: a putative spindle checkpoint kinase closely linked to cell proliferation. Cell Growth Differ 1998;9:877-85.

Pardee AB. A restriction point for control of normal animal cell proliferation. Proc Natl Acad Sci U S A 1974;71:1286-90.

Shedden K, Cooper S. Analysis of cell-cycle-specific gene expression in human cells as determined by microarrays and double-thymidine block synchronization. Proc Natl Acad Sci U S A 2002a;99:4379-84.

Shedden K, Cooper S. Analysis of cell-cycle-specific gene expression in Saccharomyces cerevisiae as determined by Microarrays and Multiple synchronization methods. Nucleic Acids Res 2002b;30:2920-9.

Thornton M, Eward KL, Helmstetter CE. Production of minimally disturbed synchronous cultures of hematopoietic cells. Biotechniques 2002;32: 1098-105. 